\title{
Analysis of antenatal and postnatal determinants which promote postpartum weight loss in obese women - An analysis from the UPBEAT trial
}

\section{Abstract}

In the UK $21 \%$ of pregnant women are categorised as obese ${ }^{(1)}$. Women who enter pregnancy with a high body mass index (BMI) are at greater risk of gestational weight gain (GWG) above that recommended by the Institute of Medicine (IOM) and are less likely to return to their pre-pregnancy weight compared to women with a healthy BMI ${ }^{(2)}$. Pregnancy can therefore alter a woman's weight gain trajectory across the life course, through retention of gestational weight gained. Given the increase in prevalence of obesity among women of reproductive age, the aim of this study was to identify antenatal and postnatal modifiable determinants associated with successful postpartum weight loss in women who participated in UPBEAT; a multi-centre randomised controlled trial comparing a lifestyle intervention of diet and physical activity to standard care during pregnancy.

710 women completed the 6-month postpartum follow-up visit, $464(65 \%)$ of which provided complete data for the analysis. Using regression analysis, we examined the relationship between postpartum weight retention (PPWR; calculated by subtracting prepregnancy weight from six-month postpartum weight) and modifiable determinants including: reported glycaemic index and smoking status at baseline (15-18 weeks' gestation), GWG within the IOM recommendations, self-reported postpartum physical activity (categorised as low, moderate or high) and mode of infant feeding (breastfed, formula or mixed). Women were excluded if they gave birth $<37$ weeks gestation or they were pregnant at the 6-month visit. Results were adjusted for age, BMI, ethnicity, socio-economic status, parity and randomisation arm.

In this cohort of obese women $52 \%$ were at or below their pre-pregnancy weight by 6 months postpartum. Overall, there was a reduction in PPWR of $-0.23 \pm 6.7 \mathrm{~kg}[-23.5$ to $23.0 \mathrm{~kg}]$. In an adjusted multiple regression model, women who breastfed for $\geq 4 \mathrm{months}$, had moderate or high levels of physical activity and appropriate GWG, were more likely to be at or below their pre-pregnancy weight by 6-months postpartum (all $\mathrm{p}<0.02$ ). In a mutually-adjusted multivariate model, for each additional factor women lost a further $-1.5 \mathrm{~kg}(95 \% \mathrm{CI}-2.3$ to $-0.68 ; \mathrm{p}<0.001)$ compared to their pre-pregnancy weight.

This study has shown that there is an incremental association with postpartum weight loss and the identified modifiable determinants. These findings support initiatives which target any or all these factors during the antenatal and postnatal periods to help support women with returning to their pre-pregnancy weight.

\section{Conflict of Interest}

There is no conflict of interest

1. National Maternity and Perinatal Audit: Clinical Report 2017. RCOG.

2. Ketterl TG et al. (2018) Matern. Child. Health J 22, 1339-1344. 